\begin{tabular}{ll} 
POS & PROCEEDINGS \\
\hline
\end{tabular}

\title{
R-evolving QCD matrix elements
}

\section{André H. Hoang,}

Max-Planck-Institut für Physik (Werner-Heisenberg-Institut), Föhringer Ring 6, 80805 München, Germany E-mail: ahoang@mppmu .mpg • de

\section{Ambar Jain}

Department of Physics, Carnegie Mellon University, 5000 Forbes Ave., Pittsburgh PA - 15213

E-mail:ambareandrew. cmu . edu

\section{Ignazio Scimemi*}

Departamento de Física Teórica II, Universidad Complutense de Madrid, 28040 Madrid, Spain E-mail: ignaziosefis.ucm.es

\section{lain W. Stewart}

Center for Theoretical Physics, Massachusetts Institute of Technology, Cambridge, MA 02139, USA

E-mail:iains@mit.edu

\begin{abstract}
$\overline{\mathrm{MS}}$, combined with dimensional regularization, is one of the most popular and practical renormalization scheme in quantum field theory. One of the drawbacks of this scheme is however that undesired infrared contributions hide in the short-distance calculations and manifest themselves as the so called renormalon ambiguities. We have found a simple way to remove these terms which retains the properties of calculation of $\overline{\mathrm{MS}}$. The subtraction requires the introduction of a new cutoff scale, $R$, that controls power divergences. The variation of the final result with $R$ is solved with an appropriate differential equation, very similar to the usual Renormalization Group equations. The evolution so defined is called R-evolution. We illustrate the application to EllisJaffe sum rule.
\end{abstract}

Preprint numbers: MPP-2010-5, MIT-CTP 4114

9th International Symposium on Radiative Corrections (Applications of Quantum Field Theory to Phenomenology)

25 - 30 October 2009, Ascona, Switzerland

\footnotetext{
${ }^{*}$ Speaker.
} 
One of the major instrument for calculations in particle physics is the operator product expansion (OPE). The program of the OPE consists in factorizing contributions which can be calculated with perturbation theory from matrix elements which need either an experimental determination or non-perturbative methods. The perturbative calculations are meaningful at an energy scale $Q$ which is much bigger then $\Lambda_{\mathrm{QCD}}$, the energy scale at which non-perturbative effects become important. In the Wilsonian OPE, the observables are factorized using a cutoff, or factorization scale, $\Lambda^{f}$, where $\Lambda_{\mathrm{QCD}}<\Lambda^{f}<Q$, and are expanded in $\Lambda_{\mathrm{QCD}} / Q$. Consider a dimensionless observable $\sigma$ whose $\mathrm{OPE}$ is

$$
\sigma=C_{0}^{W}\left(Q, \Lambda^{f}\right) \theta_{0}^{W}\left(\Lambda^{f}\right)+C_{1}^{W}\left(Q, \Lambda^{f}\right) \frac{\theta_{1}^{W}\left(\Lambda^{f}\right)}{Q^{p}}+\ldots
$$

The $C_{0,1}^{W}$ are dimensionless Wilson coefficients containing contributions from momenta $k>\Lambda^{f}$ with perturbative expansions in $\alpha_{s}$, and $\theta_{0,1}^{W}=\left\langle\mathscr{O}_{0,1}\right\rangle_{W}$ are non-perturbative matrix element with mass dimensions 0 and $p$, containing contributions from $k<\Lambda^{f} . C_{0,1}^{W}\left(Q, \Lambda^{f}\right)$ contain an infinite series of terms, $\left(\Lambda^{f} / Q\right)^{n}$, modulo $\ln ^{m}\left(\Lambda^{f} / Q\right)$ terms, and this reflects the fact that $C_{0,1}^{W}$ only include contributions from momenta $k>\Lambda^{f}$. The hard scale $Q$ is contained only in the Wilson coefficients. The Wilsonian OPE provides a separation of momentum scales, but it is difficult to define $\Lambda^{f}$, retain gauge symmetry and Lorentz invariance, and perturbative computations beyond one-loop are very difficult. The calculations are easier in dimensional regularization and the $\overline{\mathrm{MS}}$ renormalization scheme preserves symmetries almost automatically. Eq. (1) becomes

$$
\sigma=\bar{C}_{0}(Q, \mu) \bar{\theta}_{0}(\mu)+\bar{C}_{1}(Q, \mu) \frac{\bar{\theta}_{1}(\mu)}{Q^{p}}+\ldots
$$

where $\mu$ is the renormalization scale and bars are used for $\overline{\mathrm{MS}}$ quantities. In $\overline{\mathrm{MS}}$ the $\bar{C}_{i}$ are simple series in $\alpha_{s} . C_{i}^{W}\left(Q, \Lambda^{f}\right)$ and $\bar{C}_{j}(Q, \mu)$ are perturbatively related to each other, so Eqs. (1) and (2) are just the same OPE in two different schemes. The renormalization scale $\mu$ in $\overline{\mathrm{MS}}$ plays the role of $\Lambda^{f}$. This is precisely true for logarithmic contributions, $\ln \mu \leftrightarrow \ln \Lambda^{f}$, and here the Wilsonian picture of scale separation in $\bar{C}_{i}$ and $\bar{\theta}_{i}$ carries over: the Wilson coefficients contain powers of $\ln ^{n} \mu / Q$ and matrix elements powers of $\ln ^{n^{\prime}} \mu / \Lambda_{Q C D}$ such that all $\mu$-dependence is finally canceled in eq. (2). For a generic value of $\mu$ both $\ln \mu / Q$ and $\ln \mu / \Lambda_{Q C D}$ can be big. The usual solution of this problem is to resum all $\ln \mu / Q$ using renormalization group equations (RGE) so that the choice $\Lambda_{Q C D} \lesssim \mu \ll Q$ can be made and a convergent result is obtained.

The same is not true for power law dependences on $\Lambda^{f}$. The integrations in dimensional regularization are carried out over all momenta and the subtractions of $\overline{\mathrm{MS}}$ allow $\bar{C}_{i}$ to contain some contributions from arbitrary small momenta, and the $\bar{\theta}_{i}$ to have contributions from arbitrary large momenta. While this simplifies higher order computations, it leads to factorial growth in the perturbative coefficients. In $\bar{C}_{0}$, the dominant term in the coefficient of $\left[\alpha_{s}(\mu) /(4 \pi)\right]^{n}$ is $\simeq(\mu / Q)^{p} n !\left[2 \beta_{0} / p\right]^{n} Z$ at large $n$ [1], for constant $Z$. In practice this sometimes leads to poor convergence already at one or two loop order in QCD. This poor behavior is canceled by corresponding instabilities in $\bar{\theta}_{1}$, and is referred to as an order- $p$ infrared renormalon in $\bar{C}_{0}$ canceling against an ultraviolet renormalon in $\bar{\theta}_{1}[2,3,4]$. All these features are related to the fact that the $\overline{\mathrm{MS}} \mathrm{OPE}$ does not strictly separate momentum scales. 
In ref. [10] we have proposed a new method to deal with this pathological behavior of the $\overline{\mathrm{MS}}$ Wilson coefficients. The renormalization scheme so obtained is called MSR. The new renormalization scheme is characterized by a cutoff scale, $R$, at which the renormalon contribution is subtracted, and an appropriate differential equation of the new coefficient with respect to $R$. The solution of these equations allows a perturbative resummation of logs with a complete renormalon cancellation.

The subtraction mechanism works as follows. Writing the series

$$
\ln \bar{C}_{0}(Q, \mu)=\sum_{n=1}^{\infty} a_{n}(\mu / Q)\left[\frac{\alpha_{s}(\mu)}{(4 \pi)}\right]^{n}
$$

with $a_{n}(\mu / Q)=\sum_{k=0} a_{n k} \ln ^{k} \mu / Q$ we define the MSR scheme by the series [10]

$$
\ln C_{0}(Q, R, \mu) \equiv \sum_{n=1}^{\infty}\left\{a_{n}\left(\frac{\mu}{Q}\right)-\frac{R^{p}}{Q^{p}} a_{n}\left(\frac{\mu}{R}\right)\right\} \frac{\alpha_{s}^{n}(\mu)}{(4 \pi)^{n}} .
$$

This definition cancels the order- $p$ renormalon for large $n[5,10,11]$ due to the power dependence of the subtracted term. It yields also the very simple relation

$$
C_{0}(Q, R, \mu)=\bar{C}_{0}(Q, \mu)\left[\bar{C}_{0}(R, \mu)\right]^{-(R / Q)^{p}}
$$

which must be expanded order-by-order in $\alpha_{s}(\mu)$ to remove the renormalon. Thus the coefficient $C_{0}(Q, R, \mu)$ for the MSR scheme is obtained directly from the $\overline{\mathrm{MS}}$ result. Note that $C_{0}(Q, Q, \mu)=1$ to all orders. The appropriate $p$ is obtained from the $\overline{\mathrm{MS}} \mathrm{OPE}$ by $p=\operatorname{dimension}\left(\bar{\theta}_{1}\right)-\operatorname{dimension}\left(\bar{\theta}_{0}\right)$. MSR preserves gauge invariance, Lorentz symmetry, and the simplicity of $\overline{\mathrm{MS}}$.

The appropriate values for $R$ are constrained by power counting and the structure of large logs in the OPE. The power counting $\bar{\theta}_{1} \sim \Lambda_{\mathrm{QCD}}^{p}$ implies $\theta_{1} \sim \Lambda_{\mathrm{QCD}}^{p}$, so for the matrix element we need $R=R_{0} \sim \mu \gtrsim \Lambda_{\mathrm{QCD}}$ (meaning a larger value where perturbation theory for the OPE still converges), which minimizes $\ln \left(\mu / \Lambda_{\mathrm{QCD}}\right)$ and $\ln (\mu / R)$ terms in $\theta_{1}\left(R, \mu, \Lambda_{\mathrm{QCD}}\right)$. On the other hand, $C_{0}(Q, R, \mu)$ has $\ln (\mu / Q)$ and $\ln (\mu / R)$ terms, and for $R \sim \Lambda_{\mathrm{QCD}}$ no choice of $\mu$ avoids large logs. For $R=R_{1} \sim \mu \sim Q$ we can minimize the logs in $C_{0}(Q, R, \mu)$, but not in $\theta_{1}\left(R, \mu, \Lambda_{\mathrm{QCD}}\right)$. When the OPE is carried out in $\overline{\mathrm{MS}}$ this problem is dealt with using a $\mu$-RGE to sum large logs between $Q$ and $\Lambda_{\mathrm{QCD}}$. For MSR we must use $R$-evolution, an RGE in the $R$ variable [5], to sum $\log$ between $R_{1}$ and $R_{0}$. The appropriate R-RGE is formulated with $\mu=\kappa R$ and $\kappa \sim 1$ to ensure that there are no logs in the anomalous dimension. The $\kappa$ dependence is reduced order by order in perturbation theory. We will take $\kappa=1$ noting that the uncertainty from varying $\kappa$ is anyway well captured by our method for the theory error analysis. So, for $C_{0}$ and $\kappa=1$,

$$
R \frac{d}{d R} \ln C_{0}(Q, R, R)=\bar{\gamma}\left[\alpha_{s}(R)\right]-\left(\frac{R}{Q}\right)^{p} \gamma\left[\alpha_{s}(R)\right]
$$

where $\bar{\gamma}\left[\alpha_{s}\right]=\sum_{n=0}^{\infty} \bar{\gamma}_{n}\left[\alpha_{s}(R) / 4 \pi\right]^{n+1}$ and $\gamma\left[\alpha_{s}\right]=\sum_{n=0}^{\infty} \gamma_{n}\left[\alpha_{s}(R) / 4 \pi\right]^{n+1}$ are the $\overline{\mathrm{MS}}$ and $R$ anomalous dimensions respectively. Eq. (6) can be combined with the usual $\mu$-RGE

$$
\mu \frac{d}{d \mu} \ln C_{0}(Q, R, \mu)=\bar{\gamma}\left[\alpha_{s}(\mu)\right]
$$




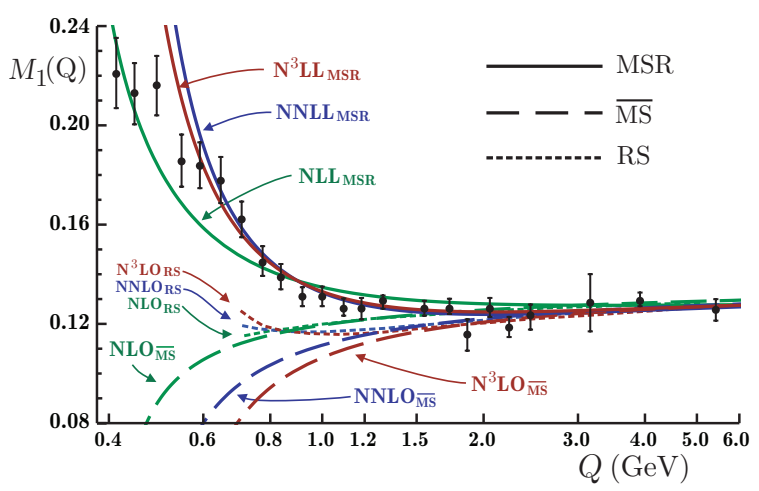

Figure 1: Perturbative results for the Ellis-Jaffe sum rule in the MSR, RS, and $\overline{\mathrm{MS}}$ schemes, at leading order in $1 / Q^{2}$. For all curves the one parameter, $\hat{a}_{0}$, is fixed by data at $Q \simeq 5 \mathrm{GeV}$.

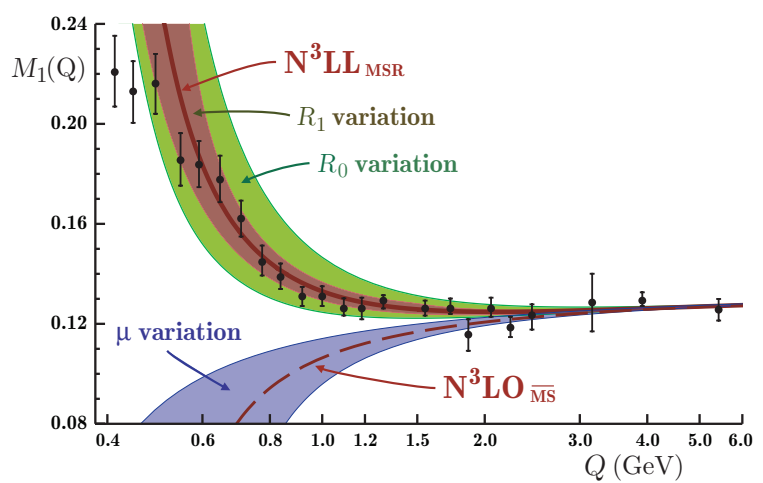

Figure 2: Uncertainty estimates in the MSR scheme and $\overline{\mathrm{MS}}$ scheme for the Ellis-Jaffe sum rule at leading order in $1 / Q^{2}$.

to resum ultraviolet (UV) logs. The fact that on the left hand side of eq. $(6,7)$ only $\ln C_{0}$ is present ensures that one can move freely in the $\mu$-R plane and that the final result depends only on the initial and final points on this plane. For $R_{1}>R_{0}$ the solution of Eq. (6) is

$$
C_{0}\left(Q, R_{0}, R_{0}\right)=C_{0}\left(Q, R_{1}, R_{1}\right) U_{R}\left(Q, R_{1}, R_{0}\right) U_{\mu}\left(R_{1}, R_{0}\right)
$$

where $U_{\mu}$ is the usual $\overline{\mathrm{MS}}$ evolution factor and $U_{R}$ is the $R$-evolution obtained by integrating the second term in eqn (6).

\section{The Ellis-jaffe sum rule in MSR}

In previous papers we have applied the principles of R-evolution to heavy quark masses and other observables $[5,10]$. Here we review the application to the Ellis-Jaffe sum rule.

In $\overline{\mathrm{MS}}$ the Ellis-Jaffe sum rule [6] for the proton in DIS with momentum transfer $Q$ is $M_{1}(Q)=$ $\left[\bar{C}_{B}(Q, \mu) \theta_{B}+\bar{C}_{0}(Q, \mu) \hat{a}_{0} / 9\right]+\bar{\theta}_{1}(\mu) / Q^{2} . \bar{C}_{B, 0}$ are known at 3 loops [7]. The two leading order terms are written so that both coefficients and matrix elements are separately $\mu$-independent: $\theta_{B}=g_{A} / 12+a_{8} / 36$ is given by the axial couplings $g_{A}=1.2694$ and $a_{8}=0.572$ for the nucleon and hyperon, while $\hat{a}_{0}$ is a $Q$ independent $\overline{\mathrm{MS}}$ matrix element. $\bar{\theta}_{1}$ denotes all $1 / Q^{2}$ power corrections with their Wilson coefficients at tree level. The $\overline{\mathrm{MS}}$ coefficients are affected by a $p=2$ 
renormalon [8], which is removed in the MSR scheme. Eq. (5) gives $[i=B, 0]$

$$
C_{i}(Q, R, R) \equiv \bar{C}_{i}(Q, R)\left[\bar{C}_{i}(R, R)\right]^{-R^{2} / Q^{2}} .
$$

With $R$-evolution the MSR OPE prediction is

$$
\begin{aligned}
& M_{1}(Q)=\left[C_{B}\left(Q, R_{1}, R_{1}\right) U_{R}^{B}\left(Q, R_{1}, R_{0}\right) \theta_{B}\right. \\
& \left.+C_{0}\left(Q, R_{1}, R_{1}\right) U_{R}^{0}\left(Q, R_{1}, R_{0}\right) \hat{a}_{0} / 9\right]+\theta_{1}\left(R_{0}, R_{0}\right) / Q^{2} .
\end{aligned}
$$

Figures 1,2 show perturbative predictions for the Ellis-Jaffe sum rule at leading power in $1 / Q^{2}$, compared with proton data from Ref. [9]. We use $\alpha_{s}(4 \mathrm{GeV})=0.2282$, and the 4-loop $\beta$ with 4 flavors. In Fig. 1, we show order-by-order results for the $\overline{\mathrm{MS}}$ scheme at $\mu=Q$, and for the resummed MSR scheme with $R_{1}=Q$ and $R_{0}=0.9 \mathrm{GeV}$. We fix $\hat{a}_{0}=0.141$ so that $\overline{\mathrm{MS}}$ and MSR both agree with the data for $Q \simeq 5 \mathrm{GeV}$. $\overline{\mathrm{MS}}$ agrees well with the data for large $Q$, but turns away at $Q \lesssim 2 \mathrm{GeV}$ and no longer converges. In contrast the MSR results still converge quickly and exhibit excellent agreement with the data over a wide range of $Q$ 's. The NLL MSR result already has the right curvature and, at NNLL and $\mathrm{N}^{3} \mathrm{LL}$ the agreement for $Q \geq 0.6 \mathrm{GeV}$ improves. We also display predictions in the RS scheme with subtraction scale $v_{f}=1.0 \mathrm{GeV}$ from Fig.3d of Ref. [12], which improve slightly over the $\overline{\mathrm{MS}}$ results, but may not be capturing the dominant power law dependence on the factorization scale. In Fig. 2 we show uncertainties for three loop results in the $\overline{\mathrm{MS}}$ and MSR schemes. The dashed red curve is the $\overline{\mathrm{MS}}$ prediction, and the blue band estimates the higher-order perturbative uncertainties varying $\mu$ in the range $\mu^{\min }(Q)<\mu<2 Q$. For $Q>1.5 \mathrm{GeV}, \mu^{\text {min }}=Q / 2$, while for $Q<1.5 \mathrm{GeV}, \mu^{\min }=1.3 Q /(1.1+Q /(1 \mathrm{GeV}))$. The red solid line is the MSR prediction, the red band is the perturbative uncertainty from varying $R_{1}$ in the same range as was done for $\mu$ in $\overline{\mathrm{MS}}$, and the green band estimates the $1 / Q^{2}$ power correction by varying $R_{0}=0.7$ to $1.2 \mathrm{GeV}$. Fig. 2 implies $-0.01 \mathrm{GeV}^{2} \lesssim \theta_{1}\left(R_{0}, R_{0}\right) \lesssim 0.01 \mathrm{GeV}^{2}$ in MSR, which is a much smaller power correction than the $\sim 0.1 \mathrm{GeV}^{2}$ estimate obtained from naive dimensional analysis in $\overline{\mathrm{MS}}$.

This work was supported by the EU network, MRTN-CT-2006-035482 (Flavianet), Spanish MEC, FPA2008-00592 and Ramon y Cajal Program, the Office of Nuclear Physics of the U.S. Department of Energy, DE-FG02-94ER40818, the Alexander von Humboldt foundation, and the Max-Planck-Institut für Physik guest program.

\section{References}

[1] M. Beneke, Phys. Rept. 317, 1 (1999).

[2] A. H. Mueller, Nucl. Phys. B250, 327 (1985).

[3] M. Luke et al., Phys. Rev. D51, 4924 (1995).

[4] L. Maiani et al., Nucl. Phys. B368, 281 (1992).

[5] A. H. Hoang, A. Jain, I. Scimemi, and I. W. Stewart, Phys. Rev. Lett. 101, 151602 (2008).

[6] J. R. Ellis and R. L. Jaffe, Phys. Rev. D9, 1444 (1974).

[7] S. A. Larin, T. van Ritbergen, and J. A. M. Vermaseren, Phys. Lett. B404, 153 (1997).

[8] D. J. Broadhurst and A. L. Kataev, Phys. Lett. B315, 179 (1993). 
[9] M. Osipenko et al., Phys. Rev. D71, 054007 (2005).

[10] A. H. Hoang, A. Jain, I. Scimemi and I. W. Stewart, arXiv:0908.3189 [hep-ph].

[11] A. H. Hoang, A. Jain, I. Scimemi and I. W. Stewart, in preparation.

[12] F. Campanario and A. Pineda, Phys. Rev. D72, 056008 (2005); 